\title{
Informationen der DAAO
}

Manuelle Medizin 2018 $56: 268$ https://doi.org/10.1007/s00337-0180420-7

Online publiziert: 23. Mai 2018

(c) Springer Medizin Verlag $\mathrm{GmbH}$, ein

Teil von Springer Nature 2018

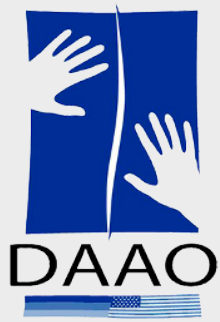

Deutsch-Amerikanische Akademie für Osteopathie DAAO e.V.

in der Deutschen Gesellschaft für Manuelle Medizin Dr. Karl-Sell-Ärzteseminar Neutrauchburg (MWE) e.V.

Riedstraße 5

88316 Isny-Neutrauchburg

Tel.: 07562-9718-0

Fax: 07562-9718-22
Dr. Oliver Drieschner (V.i.S.d.P.)

www.daao.info info@aerzteseminar-mwe.de

\section{Grundkurse}

\section{Einführungskurs}

30.08.-01.09.2018 Bad Iburg (D) SGi

Counterstrain-I-Kurs

02.09.-04.09.2018 Bad Iburg (D) PK

Muskel-Energie-Kurs

29.07.-31.07.2018 Isny (D) GKa

MFR/Lymphatics-Kurs

26.07.-28.07.2018 Isny (D) GKa

Cranio-Sacral-I-Kurs

28.06.-30.06.2018 Bad Iburg (E) LNB

01.11.-03.11.2018 Isny (E) MK

Visceral-I-Kurs

01.07.-03.07.2018 Bad Iburg (E) LNB

04.11.-06.11.2018 Isny (E) MK

BLT/Lymphatische und ligamentäre Gelenktechniken (LAS)

18.10.-20.10.2018 Isny (D) OD

Integrations-Kurs

22.06.-24.06.2018 Bad Iburg (D) SGi

21.10.-23.10.2018 Isny (D) BB

\section{Aufbaukurse}

Counterstrain-II-Kurs

23.09.-25.09.2018 Bad Iburg (E) DF

Cranio-Sacral-II-Kurs

06.07.-08.07.2018 Bad Iburg(D) RG

Visceral-II-Kur

16.09.-18.09.2018 Bad Iburg PDu

Still-FPR-Techniken

13.09.-15.09.2018 Bad Iburg (D) SGi

HVLA

20.09.-22.09.2018 Bad Iburg (E) DF

Osteopathische Woche

11.07.-15.07.2018 Isny (D) BB

28.11.-02.12.2018 Bad Iburg(D) SGi

\section{Postgraduateprogramm 2018}

Cranio-Zertifikat

Basiskurs I

29.11.-01.12.2018 München (E) Kursleiter: Prof. Michael Kuchera Basiskurs II

02.12.-04.12.2018 München (E) Kursleiter: Prof. Michael Kuchera

Membranes - Fluids - Biodynamics: a fluent concept

28.06.-30.06.2018 München (E) Kursleiter: Dr. Jason Hartmann

Osteopathy in the cranial field and practical experiences in sports injuries (including prolotherapy)

01.07.-03.07.2018 München (E) Kursleiter: Dr. Jason Hartmann

\section{Sportosteopathie-Zertifikat IV}

13.09.-15.09.2018 Köln (E) Kursleiter: Amerikanischer Kurslehrer - AOASM

Polyvagal Therapy - an Osteopathic Approach

31.08.-02.09.2018 Wien (E) Kursleiter: Prof. Michael Kuchera

Perlen in der viszeralen Osteopathie

12.10.-14.10.2018 Isny (D) Kursleiter: Dr. med. Stanley Frank

Osteopathie - Gesamtrefresher

07.11.-09.11.2018 Köln (D) Kursleiter: Dr. med.Stefan Giesswein

DAAO-Konvent

09.11.-11.11.2018 Köln (D) Kursleiter: Dr. med. Ulrich Oxfort/

Dr. med. Oliver Drieschner/

Dr. med. Jan-Raimund Schäfer

Die Kurse finden in Deutsch (D) bzw. Englisch (E) mit Übersetzungshilfe statt.

$\mathrm{U}=$ Kursleiter unbekannt.

Es sind weitere Kurstermine geplant.

Kurse in Wien und in Graz, von PCOM -Dozenten gehalten, werden auch von uns anerkannt.
Kursleiter-Legende

$\mathrm{SGi}=$ Dr. med. Stefan Giesswein $\mathrm{RG}=$ Dr. med. Rainer Galm $\mathrm{GKa}=$ Dr. med. Gert Kamjunke $\mathrm{OD}=\mathrm{Dr}$. med. Oliver Drieschner $\mathrm{BB}=$ Bärbel Bär
PK $=$ Dr. med. Petra Kramme $\mathrm{PDu}=$ Petra Duisberg LNB = Prof. Lauren Noto-Bell MK = Prof. Michael L. Kuchera DF $=$ Prof. David Fuller 